\title{
Automatic Seed Generation for Mass Extraction in Mammograms: Comparative Study
}

\author{
Ahlem Melouah
}

\begin{abstract}
For automatic breast cancer detection, mass segmentation is and continues to be a major challenge. The segmentation objective is to separate the mass from the rest of the breast by trying to delimit its borders correctly. Seeded Region Growing technique is very attractive for medical image segmentation by involving the high-level knowledge of image components in the seed selection procedure. This algorithm starts by a seed point selection and, grows seed area by exploiting the fact that pixels which are close together have similar features. In region growing process the choice of the seed point is very crucial because the overall success of the segmentation is dependent on it. In this paper, we present a comparative study of two automatic seed selection methods for breast tumor detection using seeded region growing segmentation. The first method is based on edges extraction technique; the second method is based on features extraction technique. Our results showed that seed selection method based on features extraction technique is better than seed selection method based on edges extraction technique.
\end{abstract}

Keywords - mammograms, mass detection, seed selection, segmentation, region growing.

\section{INTRODUCTION}

Breast cancer is the most common illness among women and its incidence is rising in recent years. Although the primary prevention is not completely possible since the cause of this disease is not clearly understood but, its treatment can be very effective if the breast cancer is detected in its early stages. Mammography is one of the most powerful techniques for imaging breast structures in human body. It is also the most important diagnostic tools for early breast cancer detection.

Because mammograms suffer from the poor quality caused by speckle noise and low contrast, it takes considerable effort for radiologists to extract the contours of lesions. Consequently, there is a great need for Computer-Aided-Detection system (CAD) to assist the experts for easier and better masses identification in mammograms.

Image segmentation plays a crucial role in CAD system by facilitating the delineation of the masses. Though researchers introduced several images segmentation methods but, seeded region growing (SRG) technique appears as the natural choice in the masses segmentation [1].

Seeded region growing algorithm is an automated segmentation method in which the region of interest begins as a single pixel and grows based on surrounding pixels with similar

Ahlem Melouah is with Assistant Professor, Department of Informatics, LRI labo Badji-Mokhtar Annaba University, Algeria properties. Though conventional seeded region growing is an excellent pixel-based segmentation method, unfortunately, it presents serious drawbacks. The performance of the segmentation algorithm often depends on the initial seed pixel. A correct seed pixel choice is the basic requirement for promising segmentation results. It is difficult to achieve an appropriate seed pixel because it requires an experienced user in application field both algorithm and knowledge. In order to overcome this difficulty, many automatic seed selection methods exist but, still now seeded region growing algorithm suffers from the problem of automatic seed generation [2],[3].

In this paper, two automatic seed selection methods are tested and compared. The first method based on edges extraction technique is proposed by Pohle et al. [4] and involved by Deboeverie et al. [5]. The second method based on features extraction is proposed by Yuvaria et al. [6]. The same data and the same criteria have been used in this evaluation.

This paper is organized as follows: Section 2 describes a background on the seeded region growing principal, the previous works done on automatic seed selection approaches and, a description of the experimented methods. Section 3 shows experiment results and discussion. Section 4 draws conclusions

\section{BACKGROUND}

The seeded region growing algorithm is widely used in medical images today because it effectively segments different types of images [7].

Seeded region growing proposed by Adams and Bischof [8] is based on the following principles: suppose that we start with a seed pixel located somewhere inside the suspected lesion and wish to expand from that pixel to fill a coherent region. Local pixels around the seed are examined to determine the most similar ones. So the next 4- or 8-neighboring pixels are checked for similarity so that the region can grow. Therefore, the region continues to grow until there are no remaining similar pixels among the 4- or 8-neighbors.

The overall success of the seeded region growing segmentation is dependent on seed point: If the seed point is selected outside the region of interests (ROIs), the final segmentation result will be definitely incorrect [9]. Usually, most seeded region growing methods left the seed point selection manual. However, appropriate seed position selection can be difficult to achieve and requires a user experienced with the algorithm and knowledge of the application field. To solve this problem, some seeded region growing methods discussed 
how to select a seed point automatically; these works can be classified on three categories:

- Works based on region extraction approach: the principle of these works is the extraction of the regions by using a segmentation techniques then the selection of seeds inside these regions.

- Works based on features extraction approach: these works use two steps. In the first step, region features are calculated. Generally, a region is just a window placed on the image. In the second step, a test is performed: if region features values match with some fixed features values a seed is placed in the region.

- Works based on edges extraction approach: these works applied edge detection techniques like gradient calculation, active contour and so on, to prepare seed point choice.

TABLE I. AUTOMATIC SEED SELECTION APPROACHES

\begin{tabular}{llll}
\hline \hline \multicolumn{1}{c}{ Authors } & Year & Cat. & \multicolumn{1}{c}{ Applied on } \\
\hline Shan et al. [10] & 2008 & region & $\begin{array}{c}\text { Breast ultrasound } \\
\text { images }\end{array}$ \\
\hline Mustafa et al. [11] & 2009 & region & ThinPrep ${ }^{\circledR}$ images \\
\hline Al-Faris et al. [12] & 2011 & region & Breast MRI images \\
\hline Mohd Saad et al. [13] & 2012 & region & Brain images \\
\hline $\begin{array}{c}\text { Poonguzhali and } \\
\text { Ravindran [14] }\end{array}$ & 2006 & edges & Ultrasound images \\
\hline Wu et al. [15] & 2009 & edges & abdominal MR images \\
\hline Meenalosini et al [16] & 2012 & edges & Mammograms \\
\hline $\begin{array}{l}\text { Yuvaraj and } \\
\text { Ragupathy [6] }\end{array}$ & 2013 & edges & Mammograms \\
$\begin{array}{c}\text { Pohle and Toennies } \\
\text { [4] }\end{array}$ & 2001 & features & Medical images \\
\hline Alattar et al. [17] & 2010 & features & Myocardial MR images \\
\hline \hline
\end{tabular}

A summary of automatic seed selection works done by the researchers is listed below in Table 1. Among these works those proposed by Pohel et al. [4] and Yuvarai et al. [6]. Pohel et al. exposed an automatic seed selection method base on edges extraction technique. Yuvaria et al. developed an automatic seed selection method based on features extraction approach. In the following we detail the description of these two methods.

\section{A. Seed Selection method based on Features Extraction (SSFE)}

To detect mass in mammogram using SRG segmentation, Yuvarai et al. [6] proposed a new seed point selection method based on features similarity. Statistical features like mean, dissimilarity, sum average, sum variance and auto correlation are considered as significant features able to identifier a masse. These features are computed and fixed for masses previously identified by an expert. Seed selection process starts by initializing a mask then calculate its features from the regions within the mask. If mask features did not match with the masse predefined features then the mask is shift. Otherwise, the initial pixel of the mask is taken as seed point.

\section{B. Seed Selection method based on Edges Extraction (SSEE):}

Pohle et al. [4] take the smallest gradient value as a seed point. Deboeverie et al. [5] added another contrainst: seed must have local gray value extrema. So seed pixels are automatically chosen as local gray value extrema of the image and where the gradient remains small.

\section{EXPERIMENTS AND DISCUSSION}

Image segmentation is a blind task, and there have been lots of researches to guide segmentation in a way that results give better precision of region of interest. Among segmentation algorithms, seeded region growing highly depends on where the growing process starts the accurate seed selection is very important for image segmentation [18]. In this paper we have implemented, tested and compared two automatic seed selection methods: SSFE method and SSEE method.

\section{A. Image Dataset}

For this work the MiniMIAS database provided by the Mammographic Image Analysis Society (MIAS) was used. This database which is very well-known in this area, and usually used by researchers to evaluate their algorithms, contains left and right breast images for a total of 161 patients. MiniMIAS consists of a variety of normal mammograms as well as mammograms with different characteristics and several abnormalities. The mammograms are digitized at a resolution of 1024 X 1024 pixels and at 8-bit grey scale level. All the images include the locations of any abnormalities that may be present.

\section{B. Test and discussion}

Seeded region growing has the advantage of specifying just one interested region by placing a seed inside it. Considering the fact that the selection of this seed has great influence upon the final segmentation accuracy, we propose a comparative study to verify which automatic seed selection method (SSFE or SSEE) ensure that each seed selected is indeed located inside the region of interest. The behavior of the two methods was examined, by using MiniMIAS database. We notify that, in seeded region growing segmentation process, the same similarity measure and the same threshold value is used for the two methods.

In order to compare the two considered methods (SSFE and SSEE) a seed placement criterion is adopted. This criterion consists of computing the number of correct seed placement among all tested images. 48 mammograms with tumors were taken randomly from MiniMIAS database for this comparison. The two methods are applied on each test image and, from the obtained result, we verify if the method generate a correct seed position. A seed position is considered correct if and only if it is inside the mass.

In the figure 1, two seed generation examples by the two considered methods are showed.

The first line of Figure 1 presents the first example. The original image is illustrated in Figure 1(a). Figures 1(b) shows seed generation result and segmentation result using the SSFE method. Figures 1(c) shows seed generation result and segmentation result using the SSEE method. For this example, it is visible that the SSFE method success and the SSEF method fails. The SSFE method places the seed correctly inside the mass but, the SSEE method places the seed outside the mass. In the second example (second line), the original image is illustrated in Figure 1(d). Figures 1(e) shows seed generation result and segmentation result using the SSFE method. Figures 1(f) shows seed generation result and segmentation result using the SSEE method. The second example shows an opposite result compared to the first example. The SSEE method places 
correctly the seed inside the mass but, the SSFE method places the seed outside the mass.

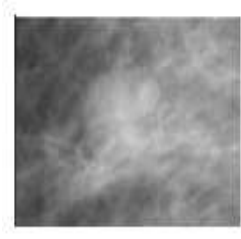

(a)

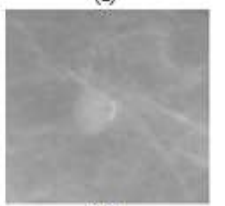

(d)

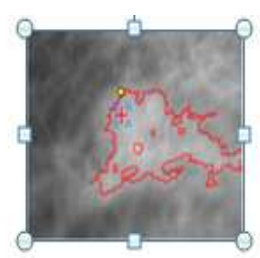

(b)

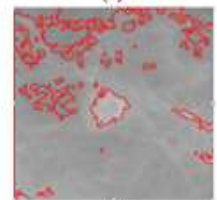

(e)

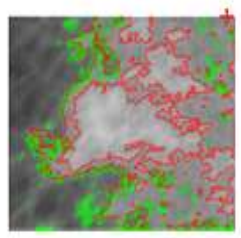

(c)

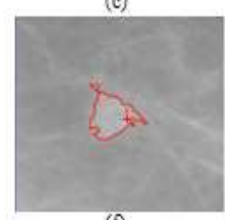

(f)
Fig 1. Seed point generation examples on two mammograms. (a, d) Original image. (b, e) Seed generation result and segmentation result using SSFE method. (c, f) Seed generation result and segmentation result using SSEE method

Among all obtained results, represented by the plot of the Figure 2, we conclude that the SSFE method is better than the SSEE method. Effectively, the SSFE method has success in over 50\% from all tested images. But, the SSEE method obtains less than $10 \%$ of correct seed placement.

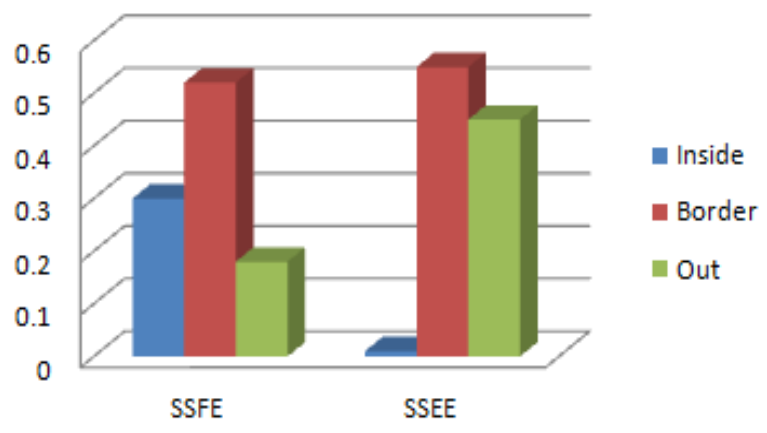

Fig 2. Statically results of SSFE and SSEE on tested mammograms

In summing up, the SSFE method can get a better seed selection result as long as the features of regions of interests match with the predefined features. Otherwise, the seed selection results can be incorrect. Note that it is very hard and expensive in time to fix, in advance, predefined features values. The big advantage of the SSFE method is its robustness against noise. In another hand, the quality of the images deeply affects the accuracy of the SSEE method. The performance of the SSEE method decrease when images noise increase. That's while the SSEE method fails with mammograms.

\section{CONCLUSION}

Today seeded region growing algorithm is widely used in medical images. Because seeded region growing result is sensitive to the initial seed, the accurate seed selection is very important for image segmentation.
In this work we have implemented, tested and evaluated two automatic seed selection methods. The first method SSFE proposed by Yuvaria et al. is based on features extraction approach. The second method SSEE proposed by Pohle et al. and involved by Deboeverie et al. is based on edge extraction approach.

The tests were elaborated on dataset randomly selected from MiniMias database. The SSFE method deals well for all tested images but, the SSEE method fails for most of them. However, additional tests must be added to improve the obtained results.

\section{REFERENCES}

[1] Mencattini A., Rabottino G., Salmeri M., Lojacono R.and Colini E. (2008) "Breast mass segmentation in mammographic images by an effective region growing algorithm", Lecture Notes in Computer Science (Springer-Verlag), Vol. 5259, pp. 948-957, October 2008. http://dx.doi.org/10.1007/978-3-540-88458-3_86

[2] Mehnert, A. and Jackway P. (1997)'An improved seeded region growing algorithm', Pattern Recognition letters, Vol 18 No.10, pp. 1065-1071. http://dx.doi.org/10.1016/S0167-8655(97)00131-1

[3] Jianping, F., Yau, D.K.Y., Elmagarmid, A.K., Aref, W.G. (2001) "Automatic image segmentation by integrating color-based extraction and seeded region growing", IEEE Trans. Image Process. Vol.10 (10), pp.1454-1466.

http://dx.doi.org/10.1109/83.951532

[4] Pohle R. and Toennies, K. D. (2001) 'A New Approach for Model-Based Adaptive Region Growing in Medical Image Analysis', Computer Analysis of Images and Patterns, Lecture Notes in Computer Science Vol. 2124, pp. 238-246 http://dx.doi.org/10.1007/3-540-44692-3_30

[5] Deboeverie, F., Veelaert, P., Philips, W. (2013) "Image segmentation with adaptive region growing based on a polynomial surface model", Journal of Electronic Imaging, Vol. 22(4), pp. 1-13. http://dx.doi.org/10.1117/1.JEI.22.4.043004

[6] Yuvaraj,K. and Ragupathy, U.S. (2013) "Automatic Mammographic Mass Segmentation based on Region Growing Technique", In 3rd International Conference on Electronics, Biomedical Engineering and its Applications (ICEBEA'2013) April 29-30, Singapore, pp 169-173.

[7] Al-Faris, A.Q. Umi Kalthum, N. MatIsa, N.A. and Shuaib, I.L. (2014) 'Computer-Aided Segmentation System for Breast MRI Tumour using Modified Automatic Seeded Region Growing, (BMRI-MASRG)", Journal Digital Imaging Vol. 27 No.1, pp 133-144. http://dx.doi.org/10.1007/s10278-013-9640-5

[8] Adams, R. and Bischof, L. (1994)'Seeded region growing', IEEE Transaction Pattern Anal. Machine Intell, Vol. 16, pp. 641-647. http://dx.doi.org/10.1109/34.295913

[9] Massich, J., Meriaudeau, F., Pérez, E., Marti, R., Oliver, A., Marti, J. (2011) "Seed selection criteria for breast lesion segmentation in Ultra-Sound images", In Workshop on Breast Image Analysis In conjunction with MICCAI, pp. 57-64.

[10] Shan, J. Cheng, HD. and Wang Y. (2008) "A novel automatic seed point selection algorithm for breast ultrasound images", In 19th International Conference on, Pattern Recognition, 8-11 Dec. 2008, Tampa, FL, pp 1-4. http://dx.doi.org/10.1109/icpr.2008.4761336

[11] Mustafa, N. Mat Isa, N.A. and Mashor, M.Y. (2009) 'Automated Multicells Segmentation of ThinPrep ${ }^{\circledR}$ Image Using Modified Seed Based Region Growing Algorithm', Biomedical Soft Computing and Human Sciences, Vol. 14 No.2, pp. 41-47.

[12] Al-Faris, A. Ngah, U.K. Isa, N.A. and Shuaib, I. (2011) 'Breast MRI Tumour Segmentation using Modified Automatic Seeded Region Growing Based on Particle Swarm Optimization Image Clustering, Soft Computing in Industrial Applications. Springer, Heidelberg, Vol 223, pp 49-60.

[13] Mohd Saad, N. Abu-Bakar, S.A.R. Muda, S. Mokji, M. and Abdullah A.R. (2012) 'Automated Region Growing for Segmentation of Brain Lesion in Diffusion-weighted MRI', Proceeding of the international MultiConference of Enginneers an Computer Scientists, vol. 1, IMECS, 14-16 March 2012, Hong Kong 
[14] Poonguzhali S. and Ravindran, G. (2006) 'A complete automatic region growing method for segmentation of masses on ultrasound images', Biomedical and Pharmaceutical Engineering International Conference on, 11-14 December 2006, pp. 88-92.

[15] Wu, J. Poehlman, S. Noseworthy M. D. and Kamath, M. V. (2009), 'Texture feature based automated seeded region growing in abdominal MRI segmentation', Journal of Biomedical Science and Engineering, ,Vol. 2 No.1, pp. 1-8 http://dx.doi.org/10.4236/jbise.2009.21001

[16] Meenalosini, S. Janet J. and E. Kannan (2012a) 'A Novel Approach in Malignancy Detection of Computer Aided Diagnosis’, American Journal of Applied Sciences Vol.9 No.7, pp. 1020-1029 http://dx.doi.org/10.3844/ajassp.2012.1020.1029

[17] Alattar, M. A. Osman N. F. and Fahmy, A. S. (2010) 'Myocardial Segmentation Using Constrained Multi-Seeded Region Growing', Campilho, Aurélio and Kamel, Mohamed (Eds), Image Analysis and Recognition, Publisher: Springer Berlin / Heidelberg, , Vol. 6112, pp. 89-98 http://dx.doi.org/10.1007/978-3-642-13775-4_10

[18] Li, G.and Wan, Y. (2010), 'Adaptive Seeded Region Growing for Image Segmentation Based on Edge Detection, Texture Extraction and Cloud Mode', Proceedings of the First international conference on Information computing and applications ICICA'10, Springer-Verlag Berlin, Tangshan, China, pp. 285-292

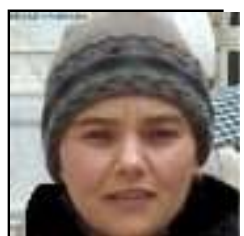

Ahlem Melouah is a Postdoctoral Research Fellow in department of Computer Science and Technology at University of Science and Technology of Annaba Algeria. In 2010, she received her Doctoral in Computer Science from University of Science and Technology of Annaba. Her research interests include medical images segmentation, medical images registration. 